No. $14(21)$

\title{
METHODS OF MATHEMATICAL QUANTUM THEORY IN SELECTED ECONOMIC MODELS
}

\section{Agnieszka Matylda Schlichtinger}

\begin{abstract}
Absract. The issue of using the physical method in economics is no longer an innovative idea. However, nowadays the methods of mathematical quantum mechanics are also applied to economic sciences. This is the natural result of the fact that as applicable in quantum mechanics, mathematical spaces and tools turn out to be useful in other fields of science. Then it is possible to assume that the problem of the choice of the space is a universal question that is associated not only with mathematics and physics but also with economics or social sciences. In this paper the author considers various formulations of Hilbert space in relation to finite-dimensional quantum mechanics which constitutes a fundament to also apply my outcomes in economics. On the basis of mathematical considerations the author puts forward the hypothesis that the complex Hilbert space is characterized with numerous advantages in relation to its real and quaternionic alternatives.
\end{abstract}

Keywords: Hilbert space, quaternions, quaternionic quantum mechanics, Schrödinger equation, regression, expected utility.

JEL Classification: B16, C02

DOI: $10.15611 / \mathrm{me} .2018 .14 .07$

\section{Introduction}

The Hilbert space constitutes a significant object of considerations in physics as well as in economics [Elsberg 1961], and even in psychology [Kahneman, Tversky 1979]. L.J. Savage [Savage 1954] is believed to be the precursor of the axiomatic approach to the problem of preferences. In the theory of Subjective Expected Utility (SEU), subjective probability distribution related to decision makers is introduced. In the paper [Eichberger, Pirner 2017], J. Eichberger and H.J. Pirner described a way of applying an abstract Hilbert space as possibility space in decision theory. The problem of applying the Hilbert space theory in econometrics also arises in Polish literature, for instance in the concept of extended regression. In the model described by

\footnotetext{
Agnieszka Matylda Schilichtinger

University of Wrocław

e-mail: mathilde.schlichtinger@gmail.com

ORCID: 0000-0002-7408-3078
} 
B. Fałda and J. Zając [Fałda, Zając 2012] regression functions are the solutions of a specified extremal problem in the finite or infinite-dimensional Hilbert space.

In the first part of this paper presents the essential problems with the formulation of quantum mechanics in the real Hilbert space, attempting to prove that only an isomorphic structure with a complex one allows for obtaining relevant results.

The second part analyzes a quaternionic quantum theory, and discusses ways of building characteristic for quantum mechanics structures using quaternionic Hilbert space.

The third part introduces the Schrödinger equation in the case of quaternionic Hilbert space.

Finally, the author compares the individual Hilbert spaces over the aforementioned numerical rings (or fields) in order to make conclusions about the advantages of the complex Hilbert space. At the same time, we notice that there are indications that the quaternionic Hilber space can introduce a new, as yet mysterious, quality into science.

From the point of view of applications in quantum mechanics, similar considerations were made in the work of Schlichtinger [2017].

\section{The real quantum theory}

The Schrödinger equation in the case of the real Hilbert space $\mathscr{H}=L_{\mathbb{R}}^{2}\left(\mathbb{R}^{3}\right)$ is considered.

In the complex Hilbert space, Stone's theorem is used to study the properties of a time evolution [Reed, Simon 1972]. The content of this theorem is as follows:

Let $U(t)$ be a strongly continuous, one-parameter group over the complex Hilbert space $\mathscr{H}$. Then there exists a self-adjoint operator $H \in \mathscr{H}$ such that

where $t$ indicates time.

$$
U(t)=e^{-i t H},
$$

According to the above, the Schrödinger equation can be obtained for a complex case. Due to the fact that the wave function $\psi(t)=U(t) \psi_{0}$, the expression takes the form:

$$
\frac{d \psi(t)}{d t}=\frac{d U(t)}{d t} \psi_{0}=\frac{d\left(e^{-i t H}\right)}{d t} \psi_{0}=-i H e^{-i t H} \psi_{0}=-i H \psi(t),
$$

where $H$ is a Hamiltonian. 
Therefore

$$
i \frac{d \psi(t)}{d t}=H \psi(t)
$$

Since $H=-i \widetilde{H}$ [Adler 1995], hence

$$
i \frac{d \psi(t)}{d t}=-i \widetilde{H} \psi(t) .
$$

In the real Hilbert space, due to the lack of imaginary unit $i$ the Schrödinger equation takes the form [Adler 1995]:

$$
\frac{d \psi(t)}{d t}=-\widetilde{H} \psi(t) .
$$

In this case the Hamilton operator $\widetilde{H}$ is a real anti-self-adjoint operator, i.e.

$$
\widetilde{H}^{*}=-\widetilde{H}
$$

Subsequently, the eigenstates of the energy operator in real quantum mechanics in finite dimensional space will be considered. The issue of the energy operator eigenvalues may be written as follows:

$$
\widetilde{H} \psi_{i}=E \psi_{i} \text {. }
$$

In the finite dimensional case, operator $\widetilde{H}$ can be presented as a real skewsymmetric matrix. The canonical form of such a matrix is [Moretti, Oppio 2016]

$$
\widetilde{H}=\left(\begin{array}{cc}
0 & -1 \\
1 & 0
\end{array}\right) \otimes D,
$$

where $\otimes$ means the Kronecker product, and $D$ is the real diagonal matrix.

Due to the fact that matrix $D$ is a real diagonal matrix, its real values will be real numbers lying on the diagonal. Therefore, the eigenvalues of operator $\widetilde{H}$ will be the products of the eigenvalues of matrix $D$ and the eigenvalues of the following matrix

$$
C=\left(\begin{array}{cc}
0 & -1 \\
1 & 0
\end{array}\right)
$$

After short transformations, the equation for the eigenvalues of the above matrix takes the form

$$
\lambda^{2}+1=0,
$$

whose only solutions are $\lambda= \pm i$. This implies that in real quantum mechanics there are no eigenstates of energy. 
Another argument that testifies to the difficulties in the formulation of quantum mechanics in real space is a certain inaccuracy that manifests itself when trying to formulate the uncertainty principle.

Let $A, B, C$ be Hermitian operators and let them fulfil the commutation relation

$$
[A, B]=A B-B A=i \hbar C .
$$

Factor $\hbar$ indicates Planck constant $h$ divided by $2 \pi$, however for the sake of clarity it can be assumed that $\hbar$ is equal to unity.

With this assumption, the above formula appears as

$$
[A, B]=A B-B A=i C \text {. }
$$

It may be noted that the commutator of two Hermitian operators is an anti-Hermitian operator:

$$
[A, B]^{*}=(A B-B A)^{*}=B^{*} A^{*}-A^{*} B^{*}=B A-A B=-[A, B] \text {. }
$$

The above fact seems to prove that the imaginary unit $i$ is a necessary element. If $C$ is a Hermitian operator by assumption, compliance can be ensured only by the existence of factor $i$.

It is possible to undertake attempts to replace imaginary unit $i$ using operator $J$ of the complex structure. However, the receipt of the uncertainty rule in such a procedure requires that $J$ satisfies the properties

i) $J^{2}=-I$,

ii) $J^{T}=-J$.

Where I means the identity operator [Moretti, Oppio 2016; Adler 1995].

Let $\mathscr{H}_{\mathrm{R}}$ be a Hilbert space over the field of complex numbers with the real scalar product. In the thus created real space, multiplication by complex scalars can be defined as follows:

$$
(a+b i) \psi=a \psi+b J \psi .
$$

The scalar product can be written as

$$
\langle\psi, \varphi\rangle_{J}=\langle\psi, \varphi\rangle+i\langle\psi, J \varphi\rangle .
$$

It is shown that the scalar product that was defined above fulfils all four properties characteristic for a complex scalar product.

1) It is demonstrated that $\langle\psi, \psi\rangle_{J}>0$ for any $\psi \neq \theta$ and that $\langle\psi, \psi\rangle_{J}=0$ if and only if $\psi=\theta$.

By definition:

$$
\langle\psi, \psi\rangle_{J}=\langle\psi, \psi\rangle+i\langle\psi, J \psi\rangle
$$


On the basis of property ii) of operator $J$ :

$$
\langle\psi, J \psi\rangle=-\langle J \psi, \psi\rangle .
$$

From the symmetry of the scalar product:

$$
\langle\psi, J \psi\rangle=\langle J \psi, \psi\rangle .
$$

It follows that

Therefore

$$
\langle J \psi, \psi\rangle=-\langle J \psi, \psi\rangle=0 .
$$

$$
\langle\psi, \psi\rangle_{J}=\langle\psi, \psi\rangle \geq 0
$$

Since $\langle\psi, \psi\rangle_{J}=\langle\psi, \psi\rangle$, hence scalar product $\langle\psi, \psi\rangle_{J}$ becomes a common scalar product, that is why $\langle\psi, \psi\rangle_{J}=0$ if and only if $\psi=\theta$.

2) The following relation is proven: $\langle\psi+\varphi, \xi\rangle_{J}=\langle\psi, \xi\rangle_{J}+\langle\varphi, \xi\rangle_{J}$.

By definition

$$
\langle\psi+\varphi, \xi\rangle_{J}=\langle\psi+\varphi, \xi\rangle+i\langle\psi+\varphi, J \xi\rangle .
$$

The right side of equality is the same as

$$
\langle\psi, \xi\rangle+\langle\varphi, \xi\rangle+i\langle\psi, J \xi\rangle+i\langle\varphi, J \xi\rangle=\langle\psi, \xi\rangle_{J}+\langle\varphi, \xi\rangle_{J},
$$

which ends the proof.

3) It is shown that $\langle\alpha \psi, \varphi\rangle_{J}=\alpha\langle\psi, \varphi\rangle_{J}$ dla $\alpha \in \mathbb{C}$.

Again, by definition:

where $\alpha=a+b i$.

$$
\langle\alpha \psi, \varphi\rangle_{J}=\langle\alpha \psi, \varphi\rangle+i\langle\alpha \psi, J \varphi\rangle
$$

Therefore

$$
\begin{aligned}
\langle\alpha \psi, \varphi\rangle+i\langle\alpha \psi, J \varphi\rangle & =\langle(a+b i) \psi, \varphi\rangle+i\langle(a+b i) \psi, J \varphi\rangle \\
& =\langle a \psi+b J \psi, \varphi\rangle+i\langle a \psi+b J \psi, J \varphi\rangle \\
& =\langle a \psi, \varphi\rangle+\langle b J \psi, \varphi\rangle+i\langle a \psi, J \varphi\rangle+i\langle b J \psi, J \varphi\rangle \\
& =a\langle\psi, \varphi\rangle_{J}+\langle b J \psi, \varphi\rangle+i\langle b J \psi, J \varphi\rangle .
\end{aligned}
$$

Using the above-mentioned properties of operator $J$, the homogeneity with respect to the first argument and the symmetry of the scalar product, it can be noted that

$$
\begin{gathered}
a\langle\psi, \varphi\rangle_{J}+\langle b J \psi, \varphi\rangle+i\langle b J \psi, J \varphi\rangle=a\langle\psi, \varphi\rangle_{J}+\langle b J \psi, \varphi\rangle+i\left\langle b \psi, J^{T} J \varphi\right\rangle \\
=a\langle\psi, \varphi\rangle_{J}+\langle b J \psi, \varphi\rangle+i\langle b \psi, I \varphi\rangle \\
=a\langle\psi, \varphi\rangle_{J}+\langle b J \psi, \varphi\rangle+i\langle b \psi, \varphi\rangle .
\end{gathered}
$$


However, by transforming the left side of equality $\langle\alpha \psi, \varphi\rangle_{J}=\alpha\langle\psi, \varphi\rangle_{J}$, one may obtain

$$
\begin{gathered}
\alpha\langle\psi, \varphi\rangle_{J}=\alpha\langle\psi, \varphi\rangle+i \alpha\langle\psi, J \varphi\rangle=(a+b i)\langle\psi, \varphi\rangle+i(a+b i)\langle\psi, J \varphi\rangle \\
=a\langle\psi, \varphi\rangle+b i\langle\psi, \varphi\rangle+a i\langle\psi, J \varphi\rangle-b\langle\psi, J \varphi\rangle \\
=a\langle\psi, \varphi\rangle_{J}+b i\langle\psi, \varphi\rangle-b\langle\psi, J \varphi\rangle .
\end{gathered}
$$

Now it is enough to show that both sides are equal.

$$
\begin{gathered}
a\langle\psi, \varphi\rangle_{J}+\langle b J \psi, \varphi\rangle+i\langle b \psi, \varphi\rangle=a\langle\psi, \varphi\rangle_{J}+b\langle J \psi, \varphi\rangle+b i\langle\psi, \varphi\rangle \\
=a\langle\psi, \varphi\rangle_{J}+b i\langle\psi, \varphi\rangle-b\langle\psi, J \varphi\rangle,
\end{gathered}
$$

which ends the proof. Anti-linearity of the scalar product in the second argument results from subsections 3 and 4 .

4) It is proven that $\langle\psi, \varphi\rangle_{J}=\overline{\langle\varphi, \psi\rangle_{J}}$.

In essence,

$$
\langle\psi, \varphi\rangle_{J}=\langle\psi, \varphi\rangle+i\langle\psi, J \varphi\rangle=\langle\varphi, \psi\rangle-i\langle\varphi, J \psi\rangle=\overline{\langle\varphi, \psi\rangle_{J}},
$$

which follows again from property ii) of operator $J$.

It turns out, therefore, that all the axioms of a complex scalar product are fulfilled for $\langle\psi, \varphi\rangle_{J}$.

The behaviour of operators in the above space is also important to consider.

Let $A$ be a self-adjoint operator in the sense of real adjointness, i.e. $A=A^{T}$. However, it is worth noting that for operator $A$ to be observable, it should commutate with operator $J$, i.e. $[A, J]=0$.

It is proved that due to the fact that the above operators are commuting, it follows that operator A must be self-adjoint in the sense of complex adjointness, i.e. $A=A^{*}$.

$$
\begin{gathered}
\langle A \psi, \varphi\rangle_{J}=\langle A \psi, \varphi\rangle+i\langle A \psi, J \varphi\rangle=\langle\psi, A \varphi\rangle+i\langle\psi, A J \varphi\rangle \\
=\langle\psi, A \varphi\rangle+i\langle\psi, J A \varphi\rangle=\langle\psi, A \varphi\rangle_{J}
\end{gathered}
$$

There is no doubt, therefore, that the above real formulation is an attempt to create a space of quantum mechanics without the use of complex numbers, but with the preservation of the most important properties of their structure.

\section{The quaternionic quantum theory}

Let $\mathscr{H}$ be the right, quaternionic Hilbert space and let $T: D(T) \rightarrow \mathscr{H}$ be right-linear operator endowed with a dense domain. An adjoint operator $T^{*}: D\left(T^{*}\right) \rightarrow \mathscr{H}$ is the only one operator that is characterized by the following properties:

i) $D\left(T^{*}\right):=\left\{u \in \mathscr{H}: \exists w_{u} \in \mathscr{H},\left\langle w_{u}, v\right\rangle=\langle u, T v\rangle \quad \forall v \in D(T)\right\}$,

ii) $\left\langle T^{*} u, v\right\rangle=\langle u, T v\rangle \quad \forall v \in D(T), \forall u \in D\left(T^{*}\right)$. 
Due to the fact that only finite-dimensional operators are considered, the above definition is reduced to the following form:

$$
\left\langle T^{*} u, v\right\rangle=\langle u, T v\rangle \forall v, u \in \mathscr{H} .
$$

If $T^{*}=T$, then such an operator is called a self-adjoint or Hermitian operator.

The issue of Hermitian operator's $T$ eigenvalue is considered.

$$
T u=u q \text {. }
$$

Therefore:

$$
q=\frac{\langle u, u\rangle q}{\langle u, u\rangle}=\frac{\langle u, u q\rangle}{\langle u, u\rangle}=\frac{\langle u, T u\rangle}{\langle u, u\rangle} .
$$

From self-adjointness of operator $T$ :

Then $q=\bar{q}$.

$$
\frac{\langle u, T u\rangle}{\langle u, u\rangle}=\frac{\left\langle T^{*} u, u\right\rangle}{\langle u, u\rangle}=\frac{\langle T u, u\rangle}{\langle u, u\rangle}=\frac{\langle u q, u\rangle}{\langle u, u\rangle}=\frac{\langle u, u\rangle \bar{q}}{\langle u, u\rangle}=\bar{q} .
$$

This means that in the quaternionic Hilbert space, the Hermitian operator has real eigenvalues, just as it does in the complex Hilbert space.

In view of the above fact, due to the alternation of quaternions with real numbers, the sphere of eigenvalues

is reduced to the form

$$
S_{q}:=\left\{\lambda^{-1} q \lambda \in \mathbb{H}: \lambda \in \mathbb{H} \backslash\{0\}\right\}
$$

$$
S_{q}:=\{q \in \mathbb{R}\} .
$$

If $u$ i $u^{\prime}$ are eigenstates of Hermitian operator $T$ and have different eigenvalues $q \neq q^{\prime}$, then:

$$
\left\langle u, T u^{\prime}\right\rangle=\left\langle u, u^{\prime}\right\rangle q^{\prime}=\left\langle T u, u^{\prime}\right\rangle=q\left\langle u, u^{\prime}\right\rangle
$$

Because the eigenvalues $q$ and $q^{\prime}$ of Hermitian operator are real, the following formula is logically correct:

which implies that

$$
\left(q-q^{\prime}\right)\left\langle u, u^{\prime}\right\rangle=0
$$

$$
\left\langle u, u^{\prime}\right\rangle=0 \text {. }
$$

Therefore, eigenstates with different eigenvalues are orthogonal. T9he spectral representation of operator $T$ appears as follows:

$$
T=\sum_{i} q_{i} P_{u_{i}}
$$

where $P_{u_{i}}(v)=u_{i}\left\langle u_{i}, v\right\rangle$ is a projection operator on state $u_{i}$.

All assuming that the eigenstates of Hermitian operator $T$ are normalized, i.e.

$$
\left\|u_{i}\right\|=1 .
$$


It can also be shown that matrix elements are of the same form as in quantum mechanics over the space of complex numbers [Moretti, Oppio 2016].

In economics, eigenvalues are present in correspondence analysis. They measure the volatility of considered variables. Eigenvalues occur in the spectral decomposition that is associated with a correspondence analysis and the principal component analysis. In the correspondence analysis [Stanimir 2005], the considered matrices are usually positive-definite. In this case eigenvalues are positive. The expansion of analysis onto a wider field may lead to complex or quaternionic eigenvalues, therefore the analysis presented above is significant in the context of economic appliance.

\section{The quaternionic Schrödinger equation}

The problem of applying Schrödinger equation to economics was considered in many papers, among others [Drabik 2011].

The study of the transformations of symmetry in quaternionic quantum mechanics may be reduced to the study of unitary quaternionic operator $U$, which is defined as follows [Reed, Simon 1972]:

$$
U^{*} U=U U^{*}=1 \text {. }
$$

In analogy to Stone's theorem, it would be conclusive if the following identity were fulfilled:

where $\widetilde{H}$ is an anti-selfadjoint operator.

$$
U=e^{\widetilde{H}},
$$

Then, definitional terms take the form:

$$
U U^{*}=e^{\widetilde{H}}\left(e^{\widetilde{H}}\right)^{*}=e^{\widetilde{H}} e^{\widetilde{H}^{*}}=e^{\widetilde{H}} e^{-\widetilde{H}}=e^{0}=I .
$$

It is also important to consider the time evolution.

It is postulated that the evolution in time is a symmetry that retains the probability. In other words, if, $\psi(t)$ and $\varphi(t)$ are two random state vectors at time $t$ and $\psi(t+\delta t)$ and $\varphi(t+\delta t)$ are respectively state vectors at the moment $t+\delta t$, then [Adler 1995]:

$$
|\langle\psi(x, t), \varphi(x, t)\rangle|=|\langle\psi(x, t+\delta t), \varphi(x, t+\delta t)\rangle| .
$$

Under the quaternion extension of Wigner's theorem, there must exist uniform quaternionic operator $U[t, \delta t]$, for which:

$$
\psi(x, t+\delta t)=U[t, \delta t] \psi(x, t) \quad \forall \psi .
$$

Expansion of terms $U[t, \delta t]$ and $\psi(t+\delta t)$ to the first row with infinitesimally small $\delta t$ and expansion coefficient $U$ that is defined as $-\widetilde{H}(t)$ leads to the formula:

$$
U[t, \delta t]=1-\delta t \widetilde{H}(x),
$$


subsequently,

$$
\psi(x, t+\delta t)=\psi(x, t)-\delta t \widetilde{H}(x) \psi(x, t) .
$$

After dividing the above equation both sides by $\delta t$ :

$$
\frac{\psi(x, t+\delta t)-\psi(x, t)}{\delta t}=-\widetilde{H}(x) \psi(x, t),
$$

where the expression on the left is identical to the definition of the time derivative from the wave function $\psi(x, t)$, one can therefore write:

$$
\frac{\partial}{\partial t} \psi(x, t)=-\widetilde{H}(x) \psi(x, t)
$$

The Schrödinger equation was obtained.

Let

where

$$
\widetilde{H}(x)=\widetilde{H}_{0}(x)+i \widetilde{H}_{1}(x)+j \widetilde{H}_{2}(x)+k \widetilde{H}_{3}(x),
$$

$$
\begin{gathered}
\widetilde{H}_{0}(x)+i \widetilde{H}_{1}(x)=\widetilde{H}_{\alpha}(x), \\
j\left(\widetilde{H}_{2}(x)-i \widetilde{H}_{3}(x)\right)=j \widetilde{H}_{\beta}(x) .
\end{gathered}
$$

Moreover, let where

$$
\psi(x, t)=\psi_{0}(x, t)+i \psi_{1}(x, t)+j \psi_{2}(x, t)+k \psi_{3}(x, t),
$$

$$
\begin{gathered}
\psi_{0}(x, t)+i \psi_{1}(x, t)=\psi_{\alpha}(x, t), \\
j\left(\psi_{2}(x, t)-i \psi_{3}(x, t)\right)=j \psi_{\beta}(x, t) .
\end{gathered}
$$

Then equation (1) takes the form:

$$
\begin{gathered}
\frac{\partial}{\partial t} \psi_{\alpha}(x, t)+\frac{\partial}{\partial t} j \psi_{\beta}(x, t) \\
=-\left(\widetilde{H}_{\alpha}(x)+j \widetilde{H}_{\beta}(x)\right)\left(\psi_{\alpha}(x, t)+j \psi_{\beta}(x, t)\right) .
\end{gathered}
$$

Equation (2) may also be represented by two equations by separating the real and quaternion parts, i.e.

$$
\begin{gathered}
\frac{\partial}{\partial t} \psi_{\alpha}(x, t)=-\widetilde{H}_{\alpha}(x) \psi_{\alpha}(x, t)+\widetilde{H}_{\beta}(x) \psi_{\beta}(x, t), \\
\frac{\partial}{\partial t} \psi_{\beta}(x, t)=-\widetilde{H}_{\beta}(x) \psi_{\alpha}(x, t)-\widetilde{H}_{\alpha}(x) \psi_{\beta}(x, t) .
\end{gathered}
$$

According to the above definitions (2), (3), $\widetilde{H}_{\alpha}(x)$ and $\widetilde{H}_{\beta}(x)$ are operators in the complex Hilbert space and $\psi_{\alpha}(x, t), \psi_{\beta}(x, t)$ (def. (4), (5)) are the wave functions in this space. Therefore, the quaternionic Schrödinger equation was presented by a system of two equations in the complex Hilbert space. 
To write the above set of equations, one may also use the two-component complex Hilbert space. The following designations are assumed:

$$
\boldsymbol{\psi}(x, t)=\left(\begin{array}{l}
\psi_{\alpha}(x, t) \\
\psi_{\beta}(x, t)
\end{array}\right) .
$$

Then equation (1) can be written as:

where

$$
\frac{\partial}{\partial t} \boldsymbol{\psi}(x, t)=-\widetilde{\boldsymbol{H}}(x) \boldsymbol{\psi}(x, t),
$$

$$
\widetilde{\boldsymbol{H}}(x)=\left(\begin{array}{cc}
\widetilde{H}_{\alpha}(x) & -\widetilde{H}_{\beta}(x) \\
\widetilde{H}_{\beta}(x) & \widetilde{H}_{\alpha}(x)
\end{array}\right) .
$$

It is proved that equation (9) is equivalent to the Schrödinger equation (2) in the quaternionic Hilbert space.

$$
\begin{gathered}
\frac{\partial}{\partial t} \boldsymbol{\psi}(x, t)=\frac{\partial}{\partial t}\left(\begin{array}{l}
\psi_{\alpha}(x, t) \\
\psi_{\beta}(x, t)
\end{array}\right)=\left(\begin{array}{l}
\frac{\partial}{\partial t} \psi_{\alpha}(x, t) \\
\frac{\partial}{\partial t} \psi_{\beta}(x, t)
\end{array}\right) . \\
-\widetilde{\boldsymbol{H}}(x) \boldsymbol{\psi}(x, t)=-\left(\begin{array}{cc}
\widetilde{H}_{\alpha}(x) & -\widetilde{H}_{\beta}(x) \\
\widetilde{H}_{\beta}(x) & \widetilde{H}_{\alpha}(x)
\end{array}\right)\left(\begin{array}{l}
\psi_{\alpha}(x, t) \\
\psi_{\beta}(x, t)
\end{array}\right) \\
=-\left(\begin{array}{l}
\widetilde{H}_{\alpha}(x) \psi_{\alpha}(x, t)-\widetilde{H}_{\beta}(x) \psi_{\beta}(x, t) \\
\widetilde{H}_{\beta}(x) \psi_{\alpha}(x, t)+\widetilde{H}_{\alpha}(x) \psi_{\beta}(x, t)
\end{array}\right) .
\end{gathered}
$$

By comparing both sides, equations (7) and (8) will be obtained.

A form that is identical to the complex Schrödinger equation may be obtained by multiplying equation (9) with imaginary unit $i$ by both sides.

$$
i\left(\frac{\partial}{\partial t} \boldsymbol{\psi}(x, t)\right)=i(-\widetilde{\boldsymbol{H}}(x) \boldsymbol{\psi}(x, t)) .
$$

The anti-selfadjointness of operator $\widetilde{\boldsymbol{H}}(x)$ implies that:

$$
-i \widetilde{\boldsymbol{H}}(x)=\boldsymbol{H}(x) .
$$

Therefore, the complex Schrödinger equation is obtained in the two-component complex Hilbert space:

$$
i \frac{\partial}{\partial t} \boldsymbol{\psi}(x, t)=\boldsymbol{H}(x) \boldsymbol{\psi}(x, t) .
$$

Because the Schrödinger quaternionic equation may be written equivalently to the two-component complex Schrödinger equation, there arises the question of whether quaternionic quantum mechanics is only a different 
way of describing complex quantum mechanics, or introducing a new quality, absent in a complex quantum mechanics.

\section{Conclusion}

Considering the relationships between real, complex and quaternionic quantum theory, it is worth noting the different approaches regarding the relevance of quaternionic Hilbert space in quantum mechanics.

In one of his works Adler [Adler 2017] puts forward the hypothesis that quaternionic quantum mechanics is a physical theory that is clearly separate from complex quantum mechanics.

Whether nature chooses a complex or a quaternionic quantum theory is ultimately an experimental matter. Many years ago, Peres proposed a test for the quaternionic effects. Without going into the intricacies of physical theories, it should be mentioned that ultimately the experiment showed some interesting properties of quaternionic quantum mechanics. It turns out that by using quaternions one can describe a specific non-commutativity of phases, which is always equal to zero in the complex quantum mechanics.

Such experiments were carried out with neutrons without result for noncommutativity of the phase. However, in his book [Adler 1995], Adler through detailed calculations revealed that quaternionic effects in certain, specified conditions [Adler 1995; 2017] contain only the commutative phase lying in the complex sub-algebra of quaternions and hence the results of neutron scattering experiments do not really give useful hints about possible quanternionic effects. Therefore, the question of whether quaternionic quantum mechanics is merely a mathematical whim, or whether it involves the structure of reality, remains open.

Returning to the discussion about the quantum mechanics formulated above, the real Hilbert space seems to be an attempt to create a space of quantum mechanics without the use of complex numbers, but maintaining the key properties of their structure. This is the premise that the structure, which is isomorphic with the field of the complex numbers, is necessary to describe the phenomena of quantum mechanics.

This is due to the fact that there are some arguments against the formulation of quantum mechanics over real space. They concern, among others, problems with the formulation of the Schrödinger equation in real space due to the lack of an imaginary unit. In real quantum mechanics there are also certain inaccuracies related to the uncertainty principle, which were discussed earlier. 
To sum up the above considerations, despite the fact that many examples seem to suggest that complex quantum mechanics is characterized by significant advantages over its real and quaternionic formulation, it is worth considering the possibility of noticing or omitting some important subtleties. It is possible that due to the fact that quantum mechanics from the beginning is developed in complex space, this formulation seems so natural that all its advantages are automatically emphasized by people studying its mathematical structure. It is also possible that there are other, unknown reasons why complex quantum mechanics seems to be optimal, however the nature of reality does not determine this at all.

In view of the above, it should be argued that the considerations presented in the work relate not only to quantum mechanics, but also to the entire nature of reality, and hence may also be useful in economic models. Physics is a science devoted to the study of reality, therefore the laws imposed by it are most likely respected by all kinds of economic mechanisms.

\section{Bibliography}

Adler S.L. (1995). Quaternionic Quantum Mechanics and Quantum Fields. Oxford University Press.

Adler S.L. (2017). Peres experiment using photons: No test for hypercomplex (quaternionic) quantum theories. Phys. Rev. A 95, 060101 (R).

Drabik E. (2011). Classical and quantum physics in selected economic models. Fundations of Management. Vol. 3. No. 1.

Eichberger J., Pirner H.J. (2017). Decision theory with a hilbert space as possibility space. Discussion Paper Series. No. 637. University of Heidelberg, Department of Economics.

Elsberg D. (1961). Risk, ambiguity, and the savage axioms. Quarterly Journal of Economics. Vol. 75, pp. 643-669.

Fałda B., Zając J. (2012). Zagadnienie regresji w naukach ekonomicznych. Metody ilościowe w badaniach ekonomicznych. Vol XIII/1, pp. 82-95.

Kahneman D., Tversky A. (1979). Prospect theory: An analysis of decision under risk. Econometrica. Vol. 47, pp. 263-291.

Moretti V., Oppio M. (2016). Quantum Theory in Real Hilbert Space, arXiv [math-ph].

Reed M., Simon B. (1972). Methods of modern mathematical physics. Vol. I. Academic Press. New York. London.

Savage L.J. (1954). Foundations of Statistics. Wiley. New York.

Schlichtinger A.M. (2017). O algebrach obserwabli w teorii kwantowej. Praca licencjacka. Uniwersytet Wrocławski.

Stanimir A. (2005). Analiza korespondencji jako narzędzie do badania zjawisk ekonomicznych. Wydawnictwo Akademii Ekonomicznej. Wrocław. 